\title{
Biocomposite nanofibrous strategies for the controlled release of biomolecules for skin tissue regeneration
}

This article was published in the following Dove Press journal:

International Journal of Nanomedicine

8 October 2014

Number of times this article has been viewed

\author{
Chinnasamy Gandhimathi' \\ Jayarama Reddy Venugopal ${ }^{2}$ \\ Velmurugan Bhaarathy ${ }^{2}$ \\ Seeram Ramakrishna ${ }^{2}$ \\ Srinivasan Dinesh Kumar ${ }^{\prime}$ \\ 'Cellular and Molecular Epigenetics \\ Laboratory, Lee Kong Chian School \\ of Medicine, Nanyang Technological \\ University, Singapore; ${ }^{2}$ Center \\ for Nanofibers and Nanotechnology, \\ Nanoscience and Nanotechnology \\ Initiative, Faculty of Engineering, \\ National University of Singapore, \\ Singapore
}

\begin{abstract}
Nanotechnology and tissue engineering have enabled engineering of nanostructured strategies to meet the current challenges in skin tissue regeneration. Electrospinning technology creates porous nanofibrous scaffolds to mimic extracellular matrix of the native tissues. The present study was performed to gain some insights into the applications of poly(L-lactic acid)-co-poly-( $\varepsilon$-caprolactone) (PLACL)/silk fibroin (SF)/vitamin E (VE)/curcumin (Cur) nanofibrous scaffolds and to assess their potential for being used as substrates for the culture of human dermal fibroblasts for skin tissue engineering. PLACL/SF/VE/Cur nanofibrous scaffolds were fabricated by electrospinning and characterized by fiber morphology, membrane porosity, wettability, mechanical strength, and chemical properties by Fourier transform infrared (FTIR) analysis. Human dermal fibroblasts were cultured on these scaffolds, and the cell scaffold interactions were analyzed by cell proliferation, cell morphology, secretion of collagen, expression of F-actin, and 5-chloromethylfluorescein diacetate (CMFDA) dye. The electrospun nanofiber diameter was obtained between $198 \pm 4 \mathrm{~nm}$ and $332 \pm 13 \mathrm{~nm}$ for PLACL, PLACL/SF, PLACL/ $\mathrm{SF} / \mathrm{VE}$, and PLACL/SF/VE/Cur nanofibrous scaffolds. FTIR analysis showed the presence of the amide groups I, II, and III, and a porosity of up to $92 \%$ obtained on these nanofibrous scaffolds. The results showed that the fibroblast proliferation, cell morphology, F-actin, CMFDA dye expression, and secretion of collagen were significantly increased in PLACL/SF/VE/Cur when compared to PLACL nanofibrous scaffolds. The accessibility of human dermal fibroblasts cultured on PLACL/SF/VE/Cur nanofibrous scaffolds proved to be a potential scaffold for skin tissue regeneration.
\end{abstract}

Keywords: poly(L-lactic acid)-co-poly-( $\varepsilon$-caprolactone), silk fibroin, fibroblast, Sirius red staining, curcumin release, skin tissue regeneration

Correspondence: Srinivasan Dinesh Kumar Cellular and Molecular Epigenetics Laboratory, Lee Kong Chian School of Medicine, Nanyang Technological University, 50 Nanyang Drive, Research Techno Plaza, X-Frontier Block, Singapore 637553

Tel +65 65923055

Fax +65 65I5 0417

Email dineshkumar@ntu.edu.sg

Jayarama Reddy Venugopal Center for Nanofibers and Nanotechnology, Nanoscience and Nanotechnology Initiative, Faculty of Engineering, National University of Singapore, Block E3, \#05-12, 2 Engineering Drive 3, Singapore II 7576 Tel +6565164272

Fax +65 68725563

Email nnijrv@nus.edu.sg

\section{Introduction}

Skin is the largest organ, covering the entire exterior of the human body, forming about $8 \%$ of the total body mass. Skin is an attractive model organ to test novel concepts of regenerative medicine, with particular emphasis on skin tissue regeneration for acute or chronic wounds. Chronic wounds seem to be a growing worldwide health and economic problem, due to the rapid increase in the number of patients with high morbidity and risk of amputations, unsatisfactory results of existing therapies, and the resulting heavy socioeconomic burden. In recent years, skin substitutes, such as xenografts, allografts, and autografts have been used for wound healing of huge dermal defects. ${ }^{1}$ Tissue engineering is a different scientific approach; the intention is to stimulate biological substitutes to change or repair the damaged organs, and is created on three main components: cells, growth factors, and scaffolds. ${ }^{2}$ Scaffolds are the main elements that interact with cells and growth factors to form neo-tissues. Therefore, several 
studies have turned in the direction of tissue-engineering techniques to promote tissue regeneration and recapture organ functions. In recent years, different types of tissueengineered skin substitutes have been developed, ${ }^{1}$ and these engineered skin substitutes have played a significant role in the repair of chronic wounds. ${ }^{2}$ One of the important factors in skin tissue engineering is the fabrication of scaffolds; the three-dimensional scaffolds deliver an extracellular matrix (ECM) analogue, which plays an essential role in host-cell penetration and physical care, to lead the proliferation and differentiation of cells to form new tissues or organs. ${ }^{3}$ Successful dermal substitutes should give a template with proper porous structure and mechanical support to grow cells, for the secretion of ECM, and to encourage angiogenesis during tissue formation for the treatment of skin deficiencies. ${ }^{4,5}$ The porous nanofibrous scaffold possesses more surface-to-volume ratio, which improves cell attachment and cell migration and helps nutrient transport to the cells more proficiently. ${ }^{6}$ The excellent mechanical properties and biodegradability of poly(1-lactic acid)-co-poly-( $\varepsilon$-caprolactone) (PLACL) do not provide a favorable surface for cell attachment and proliferation owing to insufficient cell-recognition moieties. ${ }^{7,8}$

Studies showed that the cell adhesion and proliferation could be enhanced by protein adsorption on the surface of biomaterial scaffolds. ${ }^{9,10}$ Silk fibroin (SF) is a naturally occurring protein biopolymer with several potential properties, such as biodegradability, biocompatibility, good oxygen, and water permeability, and capable of keeping aqueous surroundings for the skin, which is essential for skin tissues, and it is also used as a preservative in cosmetics. ${ }^{11-14}$ Studies proved that SF supports cell adhesion, proliferation, and differentiation; enhances collagen biosynthesis, wound healing, and re-epithelialization; and supports the improvement of atopic dermatitis and removal of scars. ${ }^{15-18}$ The exciting cytocompatibility and malleability of SF ingredients make silk an excellent biomaterial for tissue-engineering scaffolds used in the repair of many tissues, as well as skin. ${ }^{19,20}$ Previous studies showed that the electrospun vitamin $\mathrm{C}$ in combination with SF material is able to promote skin tissue regeneration. ${ }^{21}$ Studies proved that vitamin E (VE) has antioxidant, skin barrier stabilizing properties, photo-protective and anti-tumorigenic, in skin care products. $^{22,23}$ In addition to $\mathrm{VE}$, curcumin (Cur) is a naturally obtained poly-phenolic compound with excellent antioxidant, anticancer, antimicrobial, anti-inflammatory and wound-healing properties. ${ }^{24}$ Xiaoyue et al reported that the VE-loaded SF nanofibrous scaffolds enhance cell proliferation in skin fibroblast compared to pure SF scaffolds. ${ }^{25}$
In the present study, we analyze the role of SF/VE/Cur to enhance cell proliferation and the secretion of collagen for the application of skin tissue engineering.

\section{Materials and methods Materials}

Human dermal fibroblasts were obtained from the American Type Culture Collection (Manassas, VA, USA). Dulbecco's Modified Eagle's Medium (DMEM), antimycotic antibiotics, fetal bovine serum (FBS), trypan blue, and trypsin-ethylenediaminetetraacetate (EDTA) were obtained from Gibco (Thermo Fisher Scientific, Waltham, MA, USA). PLACL (70:30, Mw $150 \mathrm{kDa}$ ) was purchased from Boehringer Ingelheim Pharma, GmbH \& Co., Ingelheim, Germany. SF was obtained from Zhang Peng International Trading, Singapore. Curcumin, $\alpha$-tocopherol and 1,1,1,3,3,3-hexafluoroisopropanol (HFIP) were obtained from Sigma-Aldrich, (St Louis, MO, USA).

\section{Electrospinning of nanofibrous scaffolds}

PLACL dissolved in HFIP at 10\% (w/v); PLACL/SF solution was prepared 80:20 (w/w) at the concentration of 10\% in HFIP and PLACL/SF/VE solution was prepared 70:20:10 (w/w) at the concentration of $10 \%$ in HFIP. PLACL/SF/VE/Cur solution was also prepared at the ratio of 65:20:10:5 in HFIP at the same concentration of $10 \%$. These solutions were stirred at room temperature overnight for better distribution and homogenization. The solutions were then loaded into a $3 \mathrm{~mL}$ syringe attached to a $27 \mathrm{G} \times 1 / 2$ blunt needle with a syringe pump (KD 100 Scientific Inc., Holliston, MA, USA) at a constant flow rate of $1.5 \mathrm{~mL} / \mathrm{h}$ with a high-voltage electric field of $18 \mathrm{kV}$ (Gamma High Voltage Research, Inc., Ormond Beach, FL, USA). The electrospun nanofibers were collected on aluminum foil wrapped on a flat collector plate kept at a distance of $12-13 \mathrm{~cm}$ from the tip of the spinneret to the collector plate. The electrospun nanofibers were collected on $15 \mathrm{~mm}$ cover slips for cell culture experiments, and consequently, the nanofibers were vacuum-dried overnight to eliminate residual solvents and used for further studies.

\section{Characterization of nanofibrous scaffolds}

The morphology of the nanofibrous scaffolds was observed under a scanning electron microscope (SEM; JEOL JSM-5600LV-SEM, JEOL, Tokyo, Japan) at an accelerating voltage of $10 \mathrm{kV}$, after the samples were coated with gold using a sputter coater (JEOL JFC-1200 fine coater). To calculate the fiber diameter of nanofibers from the SEM images, $n=8$ fibers were selected at random on all the scaffolds. The fiber diameter was 
calculated by using image analysis software ImageJ (Image Java, National Institutes of Health, Bethesda, MD, USA). The mechanical strengths of electrospun nanofibrous scaffolds were analyzed using a tabletop micro-tester (Instron 3345, Norwood, MA, USA) using load cell of $10 \mathrm{~N}$ capacities. Test samples of dimension $10 \mathrm{~mm} \times 20 \mathrm{~mm}$ were tested at a cross head speed of $10 \mathrm{~mm} / \mathrm{min}$ at ambient conditions of $25^{\circ} \mathrm{C}$ and $75 \%$ humidity; the bubble point pore diameter of the nanofibrous scaffolds was determined by using a capillary flow porometer (Porous Materials Inc., Ithaca, NY, USA). The hydrophilic or hydrophobic nature of the electrospun nanofibrous scaffolds was investigated by sessile water drop contact angle analysis using video contact angle optima surface analysis system (AST products, Billerica, MA, USA). Functional group determination was carried out by Fourier transform infrared (FTIR) spectroscopy, Avatar 380 (Thermo Fisher Scientific) above the range of $400-4,000 \mathrm{~cm}^{-1}$ at a resolution of $8 \mathrm{~cm}^{-1}$.

\section{Scaffolds degradation and curcumin release}

In in vitro degradation studies, the electrospun nanofibrous scaffolds were cut $(50 \mathrm{~mm} \times 50 \mathrm{~mm})$ into small pieces. These specimens were placed in $15 \mathrm{~mL}$ of phosphate-buffered saline (PBS) solution ( $\mathrm{pH} 7.4$ ) in an incubator at $37^{\circ} \mathrm{C}$ for fixed periods of time. Every 3 days, the buffer solution was replaced from the sample. The degraded specimens were rinsed carefully with distilled water, air-dried in vacuum, and finally, the scaffolds were weighed. Percentage weight loss was calculated from the dried weight before and after degradation, using the following formula. ${ }^{26}$

$$
\text { Weight loss } \%=W_{0}-W_{\mathrm{d}} / W_{0} \times 100(\%) \text {, }
$$

where $W_{0}$ is the original weight before degradation and $W_{\mathrm{d}}$ is the dry weight after degradation. The morphological changes in the sample before and after degradation were analyzed with SEM at an accelerated voltage of $10 \mathrm{kV}$. The Cur release profile from the Cur-loaded PLACL/SF/VE nanofibrous scaffold was analyzed in PBS; the composite nanofibrous scaffold samples $(50 \mathrm{mg}$ ) were placed in a centrifuge tube, followed with the addition of $10 \mathrm{~mL}$ PBS as the release medium. Then, the centrifuge tube was kept in an incubator at $37^{\circ} \mathrm{C}$ for further study. At particular time intervals, aliquots of samples $(1 \mathrm{~mL})$ were taken from the release medium and that quantity was substituted with $5 \mathrm{~mL}$ PBS solution $\left(37^{\circ} \mathrm{C}\right.$, $\mathrm{pH}$ 7.4) for further study. Then, the amount of Cur released at different time points up to 7 days was analyzed using UV-visible spectrophotometer at $420 \mathrm{~nm}$. With the support of the calibration curve of Cur measured in the similar condition, Cur release percentage was determined and plotted as curve versus time according to this equation:

Release $(\%)=$ Released Cur/total loaded Cur $\times 100(\%) .(2)$

\section{Culture of human dermal fibroblast}

Human dermal fibroblasts were cultured in DMEM containing $10 \%$ FBS with $1 \%$ antibiotics in a $75 \mathrm{~cm}^{2}$ cell culture flask. Cells were incubated in a $\mathrm{CO}_{2}$ incubator at $37^{\circ} \mathrm{C}$ at $5 \%$ $\mathrm{CO}_{2}$, and the medium was changed twice every week. The cultured cells (passage 4) were trypsinized by trypsin-EDTA and replated after counting with trypan blue using hemocytometer. The electrospun nanofibrous scaffolds were collected on a coverslip of $15 \mathrm{~mm}$ diameter and kept in a 24-well plate with a stainless steel ring to avoid lifting of nanofibers. The scaffolds were treated under UV light for 3 hours for sterilization and again treated with 70\% ethanol for 30 minutes and rinsed thrice with PBS for 15 minutes each in order to eliminate any residual solvent and then immersed in complete medium overnight before cell seeding. The cells were seeded on the nanofibrous scaffold at a cell density of 6,000 cells/well and tissue culture plate (TCP) was used as a control for cell culture studies.

\section{Cell proliferation}

The cell proliferation was analyzed using the colorimetric MTS assay (cell titer $96^{\circledR}$ AQueous one solution; Promega, Madison, WI, USA). The reduction of yellow tetrazolium salt [3-(4,5-dimethylthiazol-2-yl)-5-(3-carboxy methoxyphenyl)2-(4-sulfophenyl)-2 $H$ tetrazolium] in MTS to produce purple formazan crystals by dehydrogenase enzymes secreted by mitochondria of live cells formed the basis of this assay. The formazan dye shows the absorbance at $490 \mathrm{~nm}$ and the quantity of formazan crystals formed was directly proportional to the number of live cells. After culturing the cells for a period of 3, 6, and 9 days, the media was discarded from the plates and the scaffolds were rinsed with PBS to eliminate dead cells. Then, the scaffolds were incubated in 1:5 ratio mixture of MTS reagent in serum-free DMEM medium for 3 hours at $37^{\circ} \mathrm{C}$ in $5 \% \mathrm{CO}_{2}$. Finally, the sample solutions were pipetted into 96-well plates and the absorbance was measured at $490 \mathrm{~nm}$ using a microplate reader (Fluostar optima, BMG LabTech, Offenburg, Germany).

\section{Cell morphology}

The cell morphology was observed using SEM. After the 6th and 9th days of cell seeding on the scaffolds to remove 
the media from 24-well plates, the cell scaffolds were rinsed twice in PBS for 15 minutes to eliminate dead cells, and then the scaffold samples were fixed with $3 \%$ glutaraldehyde. The scaffolds were again washed with deionized water and then dehydrated with increasing concentrations of ethanol $(50 \%$, $70 \%, 90 \%$, and $100 \% \mathrm{v} / \mathrm{v}$ ) for 10 minutes. Then, the scaffolds were dried with hexamethyldisilazane (Sigma-Aldrich) overnight in fume hood. Dried cellular scaffolds coated with gold in a sputter coater and the cell morphology were detected under SEM at an accelerating voltage of $10 \mathrm{kV}$.

\section{Expression of 5-chloromethylfluorescein diacetate dye}

Fluorescent dye expression was analyzed in human dermal fibroblasts using 5-chloromethylfluorescein diacetate (CMFDA), which on cleavage of its acetates by cytosolic esterase, forms a bright fluorescent CMFDA derivative. The medium was removed from the scaffolds, followed by adding $180 \mu \mathrm{L}$ of DMEM and $20 \mu \mathrm{L}$ CMFDA $(25 \mu \mathrm{M})$ to the cells on the scaffolds and incubated at $37^{\circ} \mathrm{C}$ for 2 hours. The CMFDA dye was removed and substituted by adding complete medium to incubate cells at $37^{\circ} \mathrm{C}$ overnight. Finally, the medium was removed and the cell scaffolds were rinsed with PBS, after adding serum-free medium and were viewed under an inverted Leica DM IRB laser-scanning microscope (Leica DC 300F) at $488 \mathrm{~nm}$.

\section{Secretion of collagen}

Sirius red staining was used for analyzing the secretion of collagen in the fibroblast cultured on the scaffolds. The stronger anionic dye of sulfonic acid groups reacts with the basic groups of collagen to form a red staining. This dye is used to evaluate the secretion of collagen-containing ECM through fibroblasts in culture. After 9 days of culture, the medium was removed from the TCP and the samples were fixed with $10 \%$ formaldehyde, then stained with Harris hematoxylin to differentiate the nuclei of the cells, and rinsed three times with deionized water. After that, the scaffolds were stained with Sirius red stain containing $0.1 \%$ Sirius red F3B in an aqueous solution of picric acid for 1 hour. Finally, the cells were rinsed with mild acidified water followed by $100 \%$ ethanol and observed under a Leica BM IRB microscope. Collagen was stained red color on the yellow background in the nanofibrous scaffolds.

\section{Immunofluorescence analysis}

Human dermal fibroblasts were cultured on PLACL, PLACL/ $\mathrm{SF}, \mathrm{PLACL} / \mathrm{SF} / \mathrm{VE}$, and PLACL/SF/VE/Cur nanofibrous scaffolds and TCP using DMEM for 9 days and were analyzed for the expression of F-actin protein. The cell scaffolds were fixed in ice-cold methanol for 15 minutes. After fixing, the scaffolds were rinsed in PBS for 15 minutes and incubated for 3 minutes in $0.1 \%$ Triton X-100 solution to permeabilize the cell membrane. Nonspecific binding was blocked by incubating the cells in 3\% bovine serum albumin for 60 minutes. Rhodamine-phalloidin was added in the dilution of 1:100 for 90 minutes at room temperature to stain F-actin. The scaffolds were washed thrice with PBS for 15 minutes to eliminate the excess staining. Finally, the cells were incubated with 4,6, diamino-2-phenylindole (DAPI; Thermo Fisher Scientific) in the dilution 1:5,000 for 30 minutes. The samples were removed from 24 -well plates and mounted over a glass slide using vectashield mounting medium and the actin skeleton was examined under a laser scanning confocal microscopy.

\section{Statistical analysis}

Experiments were run in triplicates and the data presented were expressed as mean \pm standard deviation. Statistical differences were determined using analysis of variance (ANOVA). Difference was considered statistically significant at $P \leq 0.05$ and $P \leq 0.001$.

\section{Results and discussion Characterization of nanofibrous scaffolds}

Electrospinning technology has been used to fabricate nonwoven nanofibrous scaffolds from biological and/or synthetic polymers and has tremendous potential in tissue-engineering applications owing to its advantage of mimicking ECM architecture. ${ }^{27,28}$ Novel cost-effective electrospun nanofibrous scaffolds are established for wound dressing and allogeneic cultured dermal substitute through the cultivation of human dermal fibroblasts for skin defects. ${ }^{29,30}$ The present study is to examine the potentials of utilizing PLACL, PLACL/SF, PLACL/SF/VE, and PLACL/SF/VE/Cur nanofibrous scaffolds for the culture of fibroblasts for skin tissue regeneration. Figure 1 showed that the scaffolds are uniform beadless nanofibrous structures and the fiber diameter of nanofibrous scaffolds obtained around $198 \pm 4$ to $332 \pm 13 \mathrm{~nm}$, respectively (Table 1). The SF, VE, and Cur in PLACL may increase the conductivity of polymer solution to reduce the diameter of the fibers to $198 \mathrm{~nm}$. Synthetic biomaterial properties have to be improved in the physical change of nanofibrous scaffolds by including proteins/peptides such as fibronectin and Arg-Gly-Asp (RGD) series, respectively, to encourage cell attachment and infiltration. ${ }^{31,32} \mathrm{SF} / \mathrm{VE} / \mathrm{Cur}$ have been 

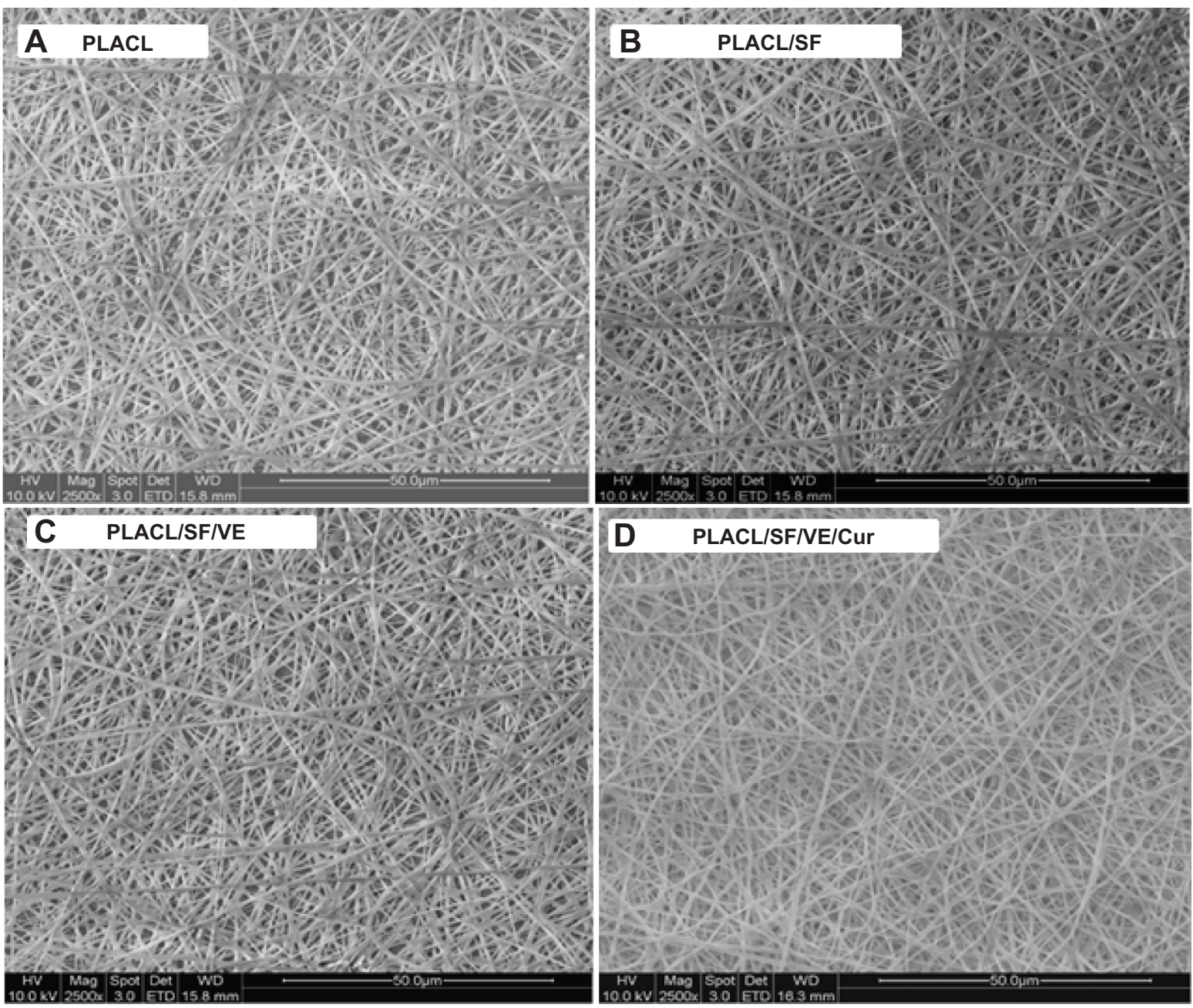

Figure I Scanning electron microscope images of the electrospun nanofibers.

Notes: (A) PLACL nanofibers, (B) PLACL/SF nanofibers, (C) PLACL/SF/VE nanofibers, and (D) PLACL/SF/VE/Cur nanofibers at 2,500× magnification (scale bar 50 $\mu$ m).

Abbreviations: PLACL, poly(L-lactic acid)-co-poly-( $\varepsilon$-caprolactone); SF, silk fibroin; VE, Vitamin E; Cur, curcumin.

commonly used as preservatives in cosmetics. ${ }^{33}$ The surface roughness of these nanofibrous scaffolds is required for cell attachment and proliferation, ${ }^{34,35}$ and is improved by the presence of functional groups and surface hydrophilicity. ${ }^{36}$ The water contact angle measurement of nanofibrous scaffolds is as shown in Table 1. The PLACL nanofibers are hydrophobic, having a contact angle of $121.2^{\circ} \pm 2.11^{\circ}$. Addition of SF improved hydrophilic properties up to $65.6^{\circ} \pm 2.78^{\circ}$. On further addition of SF and VE, the scaffold's contact angle decreases to $52.4^{\circ} \pm 1.89^{\circ}$, and with the addition of Cur, the hydrophilic value of the scaffold slightly increases to $71.3^{\circ} \pm 3.36^{\circ}$. The rate of water absorbance is directly proportional to the hydrophilic properties of the scaffolds and also supports the prevention of dehydration and exudates buildup on the wounds. ${ }^{37}$ The percentage porosity of the scaffolds was observed as $94.5 \pm 1.92,93 \pm 3.5,90.3 \pm 2.8$, and $92.9 \pm 3.4$ for PLACL, PLACL/SF, PLACL/SF/VE, and PLACL/SF/ $\mathrm{VE} / \mathrm{Cur}$, respectively, showing that the scaffolds are highly

Table I Characterization of biocomposite nanofibrous scaffolds

\begin{tabular}{lllllll}
\hline $\begin{array}{l}\text { Nanofibrous } \\
\text { construct }\end{array}$ & $\begin{array}{l}\text { Fiber } \\
\text { diameter }(\mathbf{n m})\end{array}$ & $\begin{array}{l}\text { Water contact } \\
\text { angle }\left({ }^{\circ}\right)\end{array}$ & $\begin{array}{l}\text { Porosity } \\
(\%)\end{array}$ & $\begin{array}{l}\text { Bubble point pore } \\
\text { diameter }(\mu \mathrm{m})\end{array}$ & $\begin{array}{l}\text { Young's } \\
\text { modulus (MPa) }\end{array}$ & $\begin{array}{l}\text { Tensile } \\
\text { break }(\%)\end{array}$ \\
\hline PLACL & $332 \pm 13$ & $121.2 \pm 2.11$ & $94.5 \pm 1.4$ & 1.17 & 1.51 & 250.21 \\
PLACL/SF & $280 \pm 34$ & $65.6 \pm 2.78$ & $93.6 \pm 2.5$ & 1.84 & 2.86 & 125.46 \\
PLACL/SF/VE & $223 \pm 27$ & $52.4 \pm 1.89$ & $90.3 \pm 2.8$ & 2.35 & 4.62 & 74.92 \\
PLACL/SF/VE/Cur & $198 \pm 4$ & $71.3 \pm 3.36$ & $92.9 \pm 3.4$ & 2.27 & 5.57 & 66.83 \\
\hline
\end{tabular}

Abbreviations: PLACL, poly(L-lactic acid)-co-poly-( $\varepsilon$-caprolactone); SF, silk fibroin; VE, Vitamin E; Cur, curcumin. 
porous with fine fibers indicating a large surface area to the membranes (Table 1) and the bubble point pore diameter was obtained around $1.17-2.35 \mu \mathrm{m}$. The pore size and porosity as high as $>90 \%$ is a desirable range, since large pore sizes enhance the cell-supporting ability of the scaffolds and improve cell migration and nutrient flow. ${ }^{38}$

The mechanical properties of PLACL, PLACL/SF, $\mathrm{PLACL} / \mathrm{SF} / \mathrm{VE}$, and PLACL/SF/VE/Cur nanofibrous scaffolds are shown in Table 1, and the scaffolds revealed a characteristic nonlinear stress-strain curve as shown in Figure 2. The Young's modulus obtained for PLACL, PLACL/SF, PLACL/SF/VE, and PLACL/SF/VE/Cur were 1.51 MPa, 2.86 MPa, 4.62 $\mathrm{MPa}$, and 5.57 MPa, respectively. The tensile properties of PLACL/SF, PLACL/SF/VE, and PLACL/SF/VE/Cur were higher than that of PLACL nanofibers, because the strength-to-density ratio of silk is up to ten times higher than that of steel. ${ }^{39}$ The results indicated (Table 1) that blending PLACL with SF gives better mechanical properties to the nanofibrous scaffolds. Cross linking of VE and Cur may reduce the tensile break of the nanofibrous scaffolds. For tissue engineering applications, generally scaffolds have temporary mechanical support to withstand the stresses until neo-tissue is formed. The results of tensile strength studies showed that the composite nanofibrous scaffold was highly desirable for penetrating fibroblasts inside the scaffolds for skin tissue regeneration. Mechanical stability of the scaffold plays a key role as the membrane should help cell growth and proliferation and degrade by itself as novel ECM starts regenerating to the damaged tissues. The functional groups of nanofibrous scaffolds analyzed using FTIR are as shown in Figure 3. The characteristic peak of PLACL was noticed at $1,450 \mathrm{~cm}^{-1}$, $1,760 \mathrm{~cm}^{-1}, 2,950 \mathrm{~cm}^{-1}$ on the PLACL nanofibrous scaffold. Similarly the characteristic peaks of amide I, II, and III were also noticed on the PLACL/SF/VE nanofibrous scaffold at $1,650 \mathrm{~cm}^{-1}, 1,540 \mathrm{~cm}^{-1}, 1,250 \mathrm{~cm}^{-1}$. Asymmetric and symmetric stretching vibration of $\mathrm{CH} 3$, methyl symmetrical bending and phenyl skeletal were visualized on the PLACL/SF/VE nanofibrous scaffold at 2,865 $\mathrm{cm}^{-1}$, $1,460 \mathrm{~cm}^{-1}, 1,262 \mathrm{~cm}^{-1}$. The phenolic $(\mathrm{OH})$ group, $\mathrm{C}=\mathrm{C}$ stretching of alkene and aromatic ring at $3,590 \mathrm{~cm}^{-1}$, $1,595 \mathrm{~cm}^{-1}, 1,510 \mathrm{~cm}^{-1}$ was observed in FTIR spectrum of PLACL/SF/VE/Cur nanofibrous scaffold.

\section{Scaffolds' degradation and curcumin release}

The degradation study of PLACL, PLACL/SF, PLACL/SF/ VE, and PLACL/SF/VE/Cur nanofibrous scaffolds on day 20 and 40 was analyzed by SEM and the results are shown in Figure 4. On the 40th day, PLACL/SF, PLACL/SF/VE, and PLACL/SF/VE/Cur nanofibers having distinct swelling were observed in Figure 4F-H. The results of the morphological changes of PLACL, PLACL/SF, PLACL/SF/VE, and PLACL/ $\mathrm{SF} / \mathrm{VE} / \mathrm{Cur}$ nanofibers during fiber swelling after degradation are shown in Figure 4A-H. The weight loss was caused by the degradation process, the fact that soluble oligomeric substances could be dissolved in the degradation medium from the surface of polymer by the hydrolysis of polymer chains.

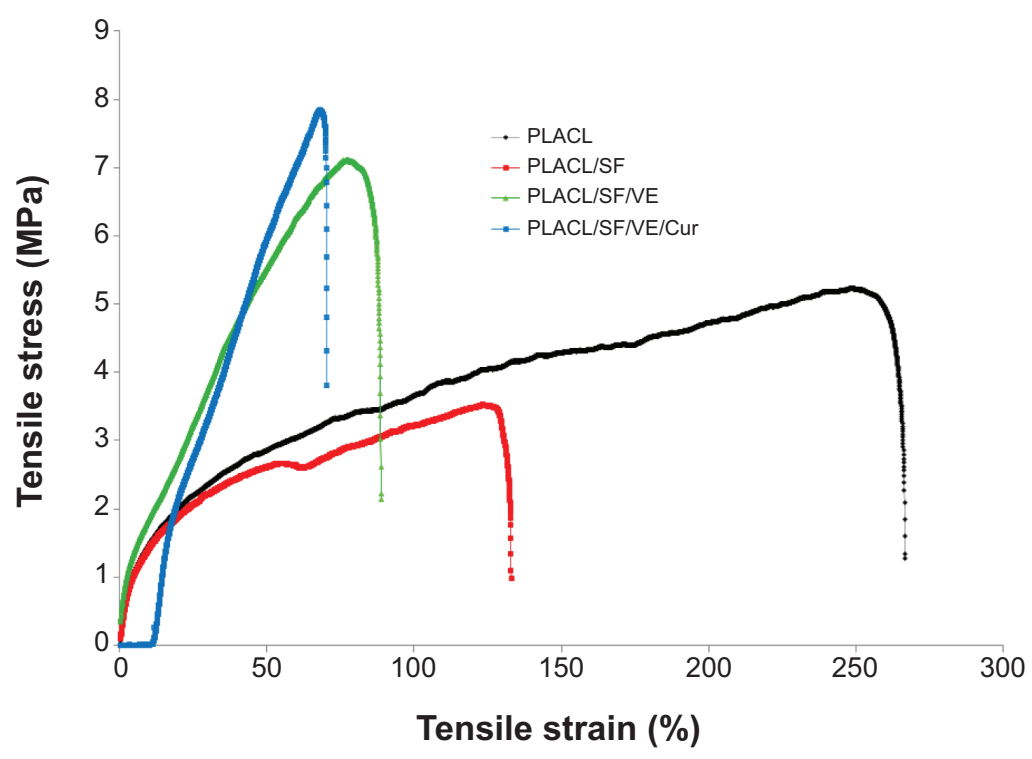

Figure 2 Tensile stress-strain curves of PLACL, PLACL/SF, PLACL/SF/VE, and PLACL/SF/VE/Cur nanofibers. Abbreviations: PLACL, poly(L-lactic acid)-co-poly-( $\varepsilon$-caprolactone); SF, silk fibroin; VE, Vitamin E; Cur, curcumin. 


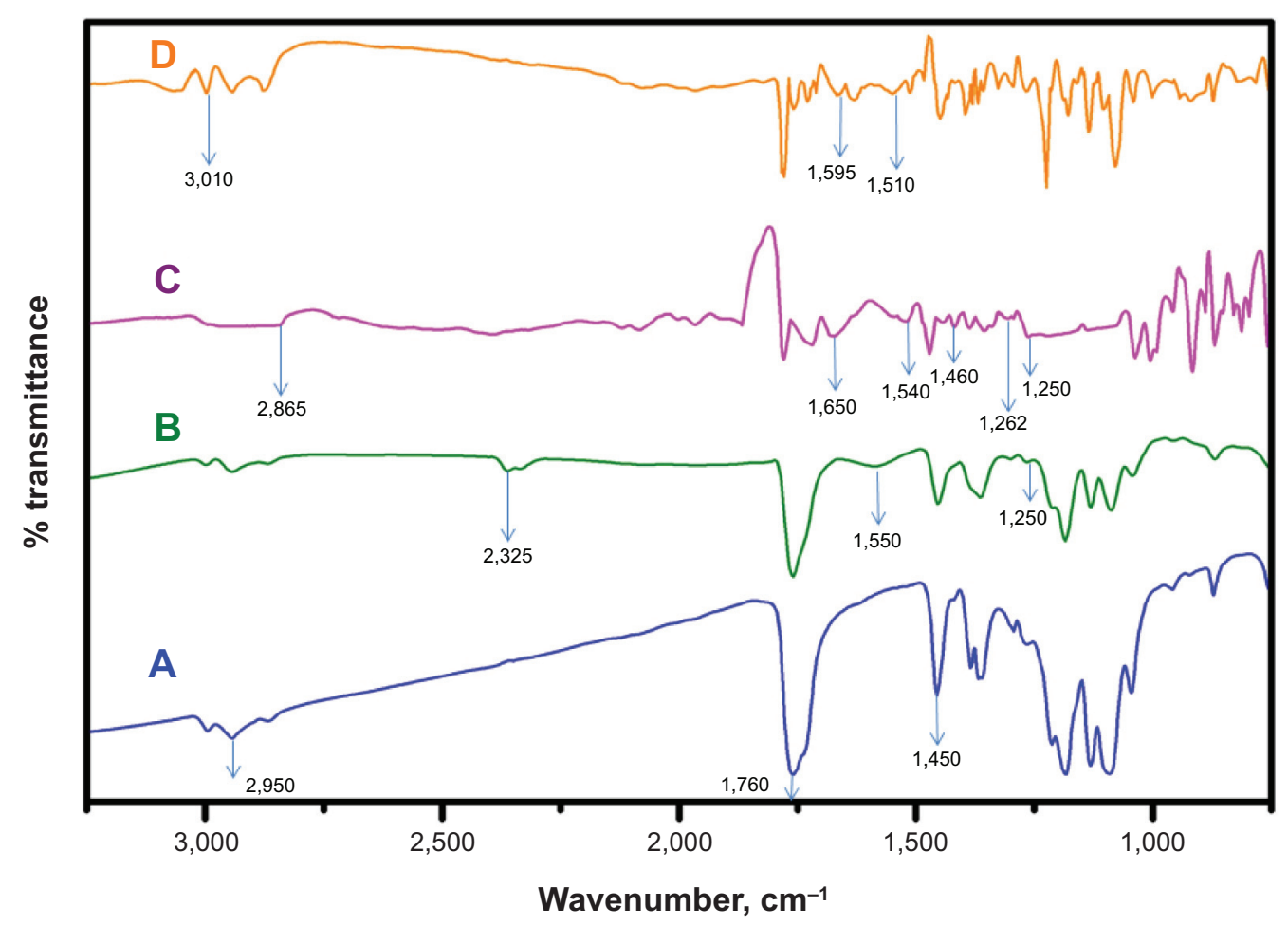

Figure 3 FTIR spectroscopic analysis of (A) PLACL, (B) PLACL/SF, (C) PLACL/SF/VE, and (D) PLACL/SF/VE/Cur nanofibers.

Abbreviations: PLACL, poly(L-lactic acid)-co-poly-( $\varepsilon$-caprolactone); SF, silk fibroin; VE, Vitamin E; Cur, curcumin; FTIR, Fourier transform infrared.

Figure $5(n=6)$ shows the weight variation of PLACL, PLACL/ SF, PLACL/SF/VE, and PLACL/SF/VE/Cur nanofibrous scaffolds from 10 to 40 days. The weight loss of PLACL nanofibrous scaffolds was $20.3 \%$ and $27.2 \%$ after degradation for 30-40 days and the degradation rate was much faster than
PLACL/SF nanofibrous scaffolds. The presence of block copolymer of poly-L-lactic acid and poly $\varepsilon$-caprolactone, PLACL composed of dissimilar chain segments, provided faster degradation level than those of poly-L-lactic acid and poly $\varepsilon$-caprolactone. ${ }^{40}$ After day 30 and 40 , and with the addition
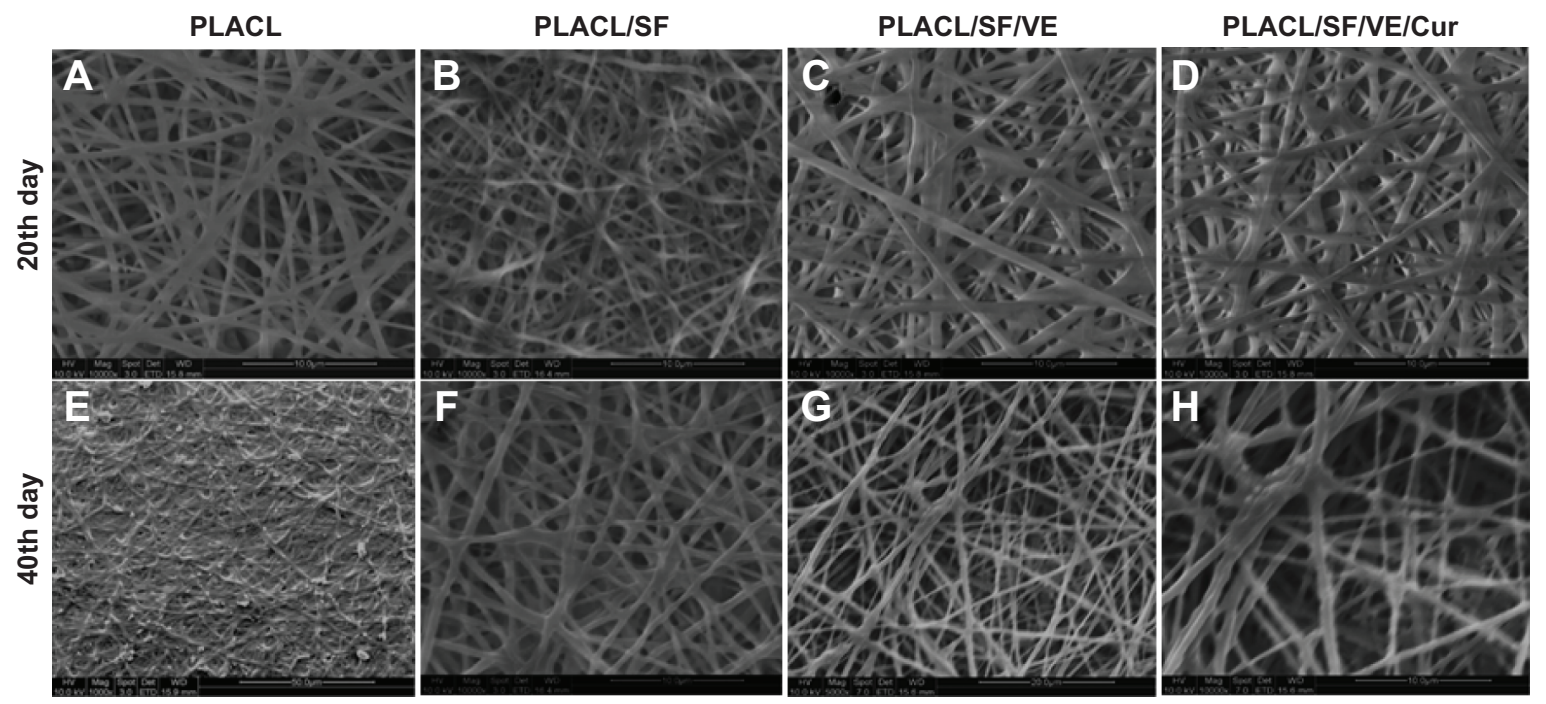

Figure 4 Degradation studies shows the SEM images of PLACL, PLACL/SF, PLACL/SF/VE, and PLACL/SF/VE/Cur nanofibrous scaffolds before and after degradation for different time in PBS.

Notes: (A) PLACL 20 days, (B) PLACL/SF 20 days, (C) PLACL/SF/VE 20 days, (D) PLACL/SF/VE/Cur 20 days, (E) PLACL 40 days, (F) PLACL/SF 40 days, (G) PLACL/SF/ VE 40 days, (H) PLACL/SF/VE/Cur 40 days.

Abbreviations: PLACL, poly(L-lactic acid)-co-poly-( $\varepsilon$-caprolactone); SF, silk fibroin; VE, Vitamin E; Cur, curcumin; PBS, phosphate-buffered saline. 


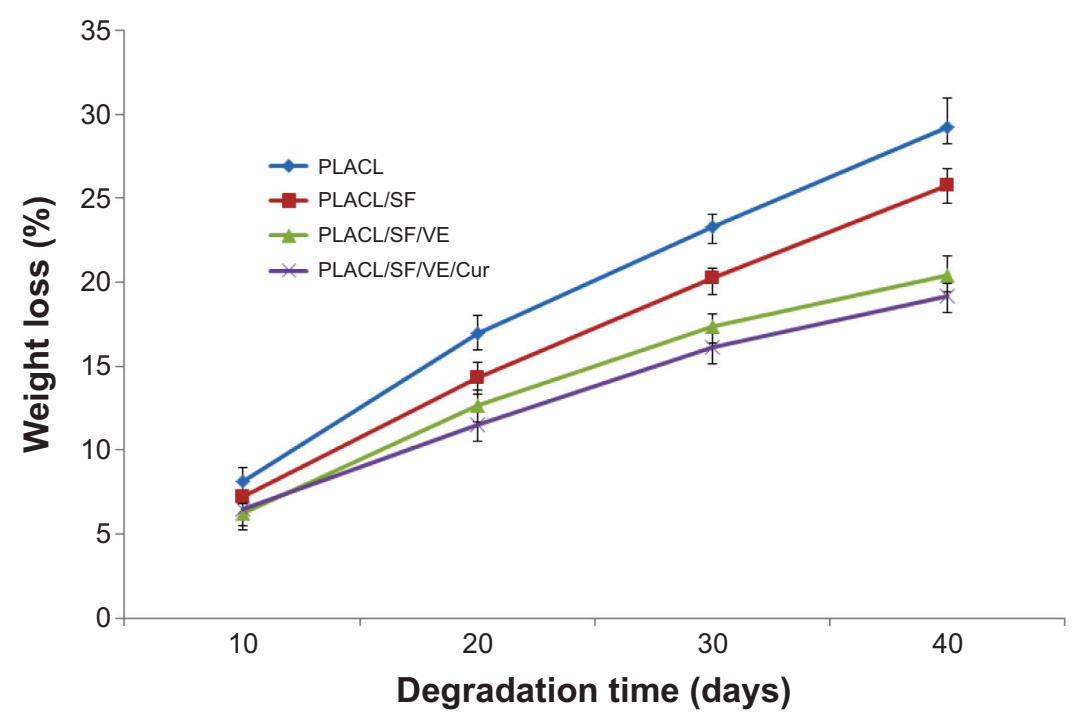

Figure 5 Changes in the dry weight of PLACL, PLACL/SF, PLACL/SF/VE, and PLACL/SF/VE/Cur nanofibrous scaffolds after degradation for different time in PBS. Abbreviations: PLACL, poly(L-lactic acid)-co-poly-(E-caprolactone); SF, silk fibroin; VE, Vitamin E; Cur, curcumin; PBS, phosphate-buffered saline.

of $20 \% \mathrm{SF}$ in PLACL/SF, PLACL/SF/VE, and PLACL/SF/ $\mathrm{VE} / \mathrm{Cur}$, the degradation rate of the nanofibrous scaffolds was reduced dramatically and the weight loss was $16 \%, 17.5 \%$, and $20 \%$ and $18 \%, 20.4 \%$, and $23 \%$, respectively. The observed results indicated that the pure PLACL nanofibrous scaffolds had the faster degradation rate when compared to PLACL/ SF, PLACL/SF/VE, and PLACL/SF/VE/Cur nanofibrous scaffolds. This was possibly owing to the intermolecular connections among SF and PLACL, which delayed movement of the PLACL molecular chains.
The in vitro drug release is an important parameter for drug delivery, the release characteristics of Cur from the nanofibrous scaffolds calculated by drug-eluting membranes of different composition, namely, $1 \%, 5 \%, 10 \%$, and $20 \%$. The release profiles of Cur from the nanofibrous scaffolds are shown in Figure 6. All release profiles revealed an initial burst release during the first 15 hours, followed by gradual release and sustained release of drugs. The maximum released percentage of Cur of $1 \%, 5 \%, 10 \%$, and $20 \% \mathrm{w} / \mathrm{w}$ Cur-loaded nanofibrous scaffolds were about $53 \%$,

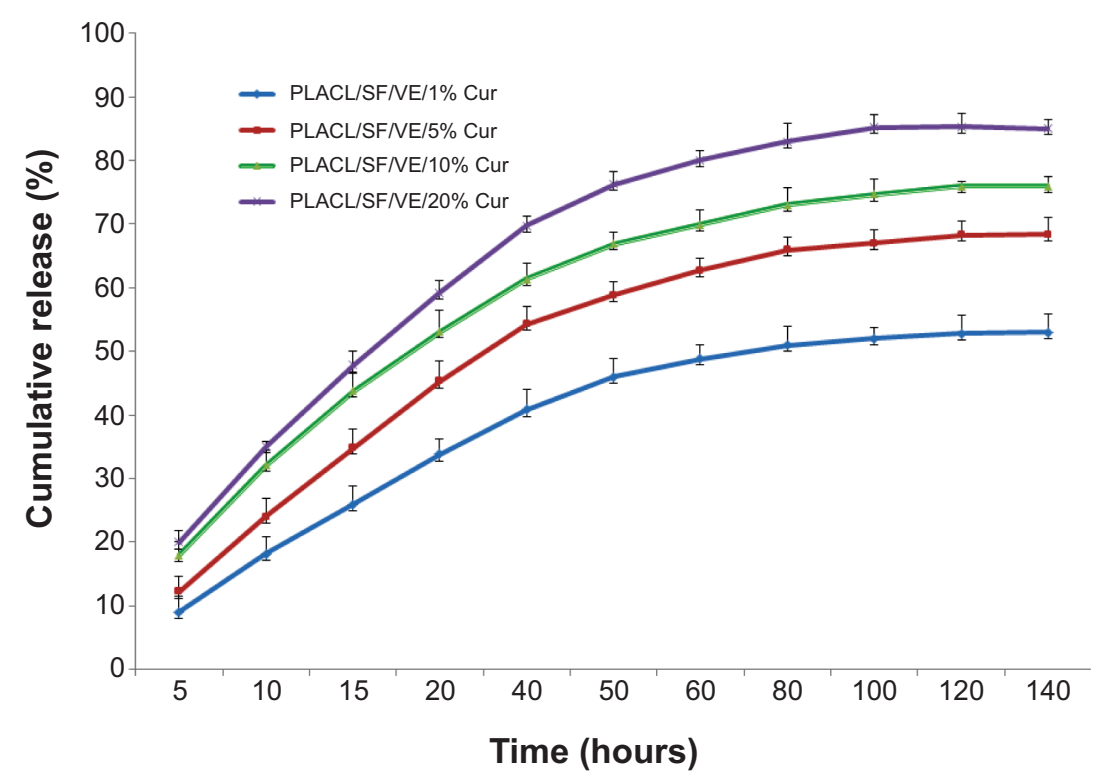

Figure 6 Cumulative release profile of curcumin from curcumin-loaded nanofibrous scaffold.

Notes: (A) PLACL/SF/VE/I\% Cur, (B) PLACL/SF/VE/5\% Cur, (C) PLACL/SF/VE/I0\% Cur, and (D) PLACL/SF/VE/20\% Cur (based on the weight of the scaffolds).

Abbreviations: PLACL, poly(L-lactic acid)-co-poly-( $\varepsilon$-caprolactone); SF, silk fibroin; VE, Vitamin E; Cur, curcumin; PBS, phosphate-buffered saline. 
$68 \%, 76 \%$, and $85 \%$, respectively. The fast release of Cur at the start time of immersion may result in the presence of solubilized Cur on the surface of the nanofibrous scaffolds. The maximum release of Cur (85\%) was obtained in $20 \%$ Cur-loaded nanofibrous scaffolds.

\section{Interaction of cells and scaffolds}

Early cell adhesion and proliferation are necessary issues in developing wound healing/dressing of nanofibrous scaffolds for skin tissue regeneration. ${ }^{41}$ The proliferation rate of fibroblasts on TCP, PLACL, PLACL/SF, PLACL/SF/ $\mathrm{VE}$, and PLACL/SF/VE/Cur nanofibrous scaffolds on day 3 , 6, and 9 was analyzed and is shown in Figure 7. The results indicated that the proliferation rate was significantly higher $(P \leq 0.001)$ in PLACL/SF and PLACL/SF/VE; and in PLACL/SF/VE/Cur when compared to TCP and PLACL nanofibers on day 6 and 9. The combination of SF, VE, and Cur to PLACL nanofibers showed that the cell proliferation rate increased significantly compared to PLACL alone on days 3, 6, and 9, indicating that SF, VE, and Cur are functionally active in stimulating cell attachment and distribution on the nanofibrous matrices. SF is a natural, biocompatible fibrous protein and possesses unique mechanical properties, containing amino acid structures that induce cell adhesion and VE shown to be mediated through increased production of interleukin-2, leading to enhanced proliferation of cells. Cur treatment resulted in an increased formation of granulation tissue, neovascularization, and enhanced biosynthesis of ECM proteins such as collagen. ${ }^{24} \mathrm{PLACL} / \mathrm{SF} / \mathrm{VE} / \mathrm{Cur}$ nanofibrous scaffolds have comparatively smaller fiber diameter of about $198 \mathrm{~nm}$ favors enhanced surface area to volume ratio and also increased oxygen and nutrients transport to the scaffolds, therefore promoting cell adhesion and proliferation for wound healing in skin.

\section{Secretion of collagen}

The wound-healing effect is determined by ECM synthesis and remodeling into a highly scheduled architecture. ${ }^{42}$ PicroSirius red staining proved ECM secretion by the cultured cells on the nanofibrous scaffolds. Figure 8 shows the collagen secretion on nanofibrous scaffolds after 9 days of cell culture. The Sirius red staining appears to be reduced in cells cultured on TCP and PLACL (Figure 8A and B) when compared to all other nanofibrous scaffolds. Furthermore, it was observed that the collagen staining was increased in PLACL/SF and PLACL/SF/VE scaffolds (Figure 8C and D) when compared to PLACL scaffolds (Figure 8B). The reason may be the capability of SF to motivate several cell signaling pathways leading to improved secretion of collagen matrix compared to PLACL scaffolds. ${ }^{43}$ Generally, PLACL biopolymers support the cell growth by adding VE; Cur induce the secretion of ECM in fibroblast cells. The results

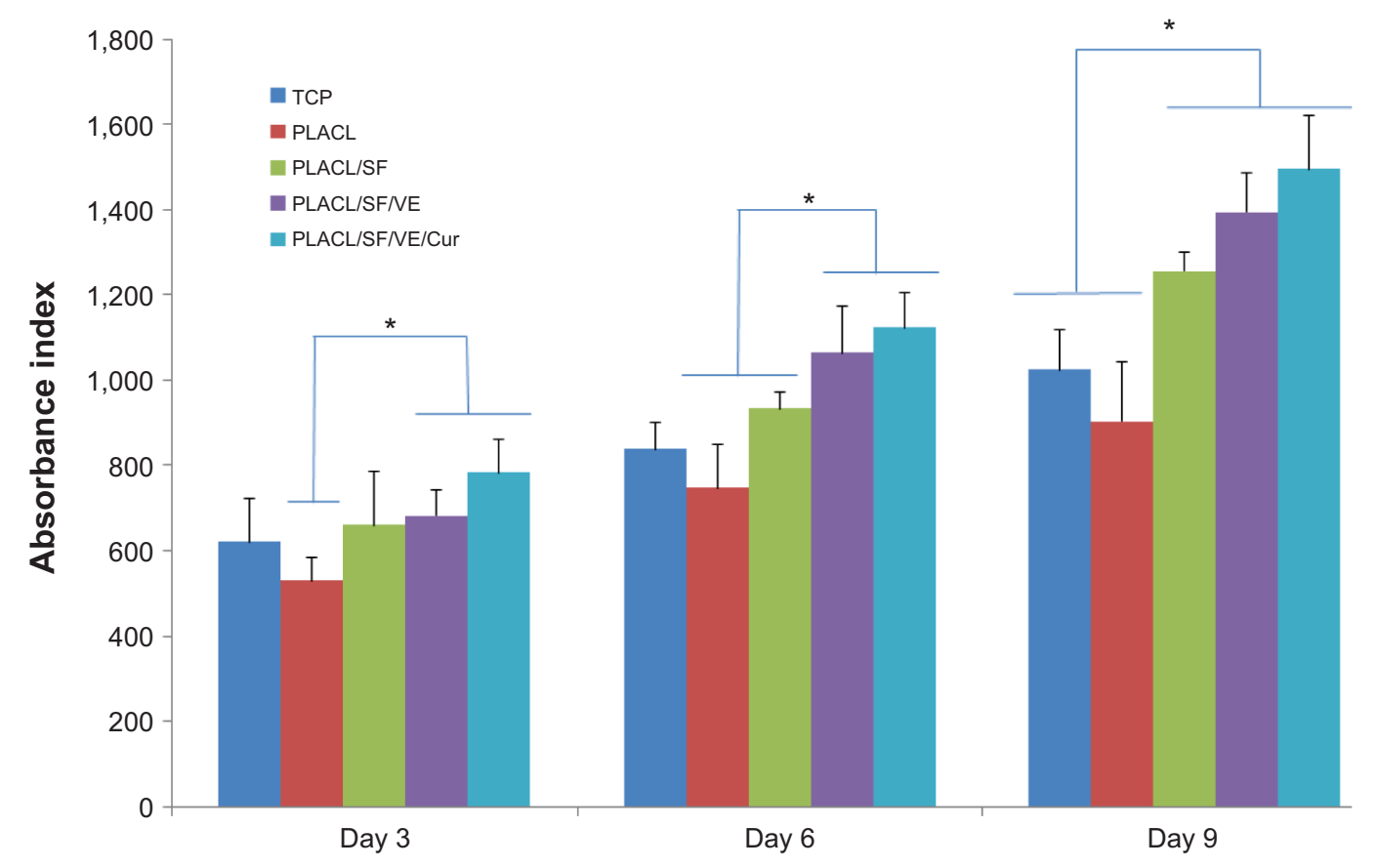

Figure 7 MTS assay for cell proliferation study on TCP, PLACL, PLACL/SF, PLACL/SF/VE, and PLACL/SF/VE/Cur nanofibers using human dermal fibroblast on day 3, 6, and 9. Notes: *Indicates significant difference of $P \leq 0.001 \quad(n=8)$.

Abbreviations: PLACL, poly(L-lactic acid)-co-poly-( $\varepsilon$-caprolactone); SF, silk fibroin; VE, Vitamin E; Cur, curcumin; TCP, tissue culture plate. 


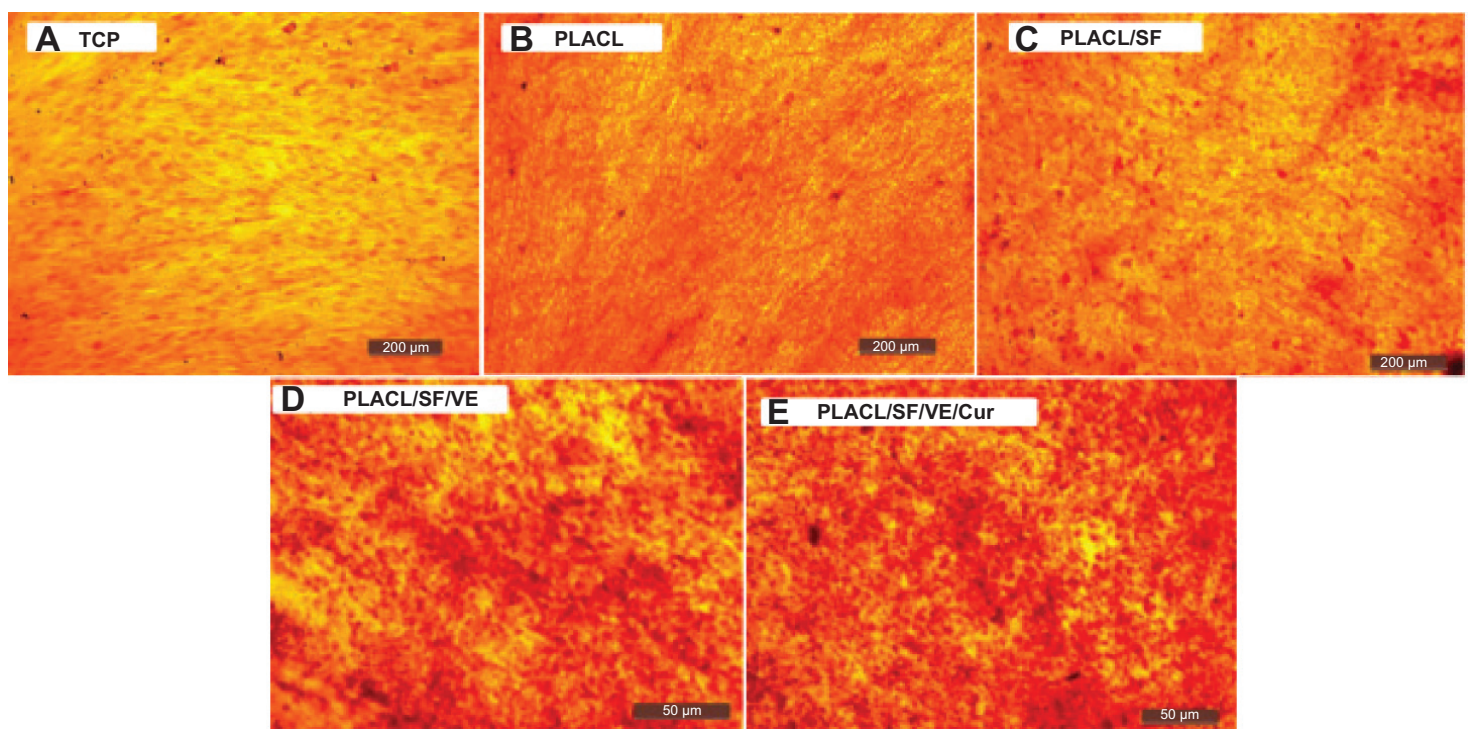

Figure 8 Sirius red staining shows the secretion of collagen by human dermal fibroblast on (A) TCP, (B) PLACL nanofibers, (C) PLACL/SF nanofibers, (D) PLACL/SF/VE nanofibers, and (E) PLACL/SF/VE/Cur nanofibers at 60× magnification (scale bar $200 \mu \mathrm{m}$ ).

Abbreviations: TCP, tissue culture plate; PLACL, poly(L-lactic acid)-co-poly-( $\varepsilon$-caprolactone); SF, silk fibroin; VE, Vitamin E; Cur, curcumin.

revealed that the collagen secretion was more on PLACL/SF/ VE/Cur (Figure 8E) nanofibrous scaffolds when compared to all other scaffolds. This abundant secretion of collagen in cells observed in PLACL/SF/VE/Cur biocomposite nanofibrous scaffolds may prove to have good potential for wound healing in skin tissue regeneration.

\section{Cell morphology}

Interaction between cells and scaffold material depends on the physical and chemical properties of the biomaterial and the predominance of chemical composition, particle size, and surface characters, which contain topography, roughness, surface energy, and hydrophilicity. ${ }^{44}$ The SEM images (Figure 9) showed normal cell morphology on TCP, PLACL, PLACL/SF, PLACL/SF/VE, and PLACL/SF/VE/ Cur scaffolds on day 6 and 9 , respectively. Compared to day 6 nanofibrous scaffolds (Figure 9A-E), on day 9 (Figure 9F-I), the cells penetrate into the nanofibrous scaffolds with improved migration and cellular connections to form a dermal substitute. The observed results indicated that the cell morphology was comparatively similar in TCP and PLACL nanofibrous scaffolds; cell-cell interaction appears to be
TCP
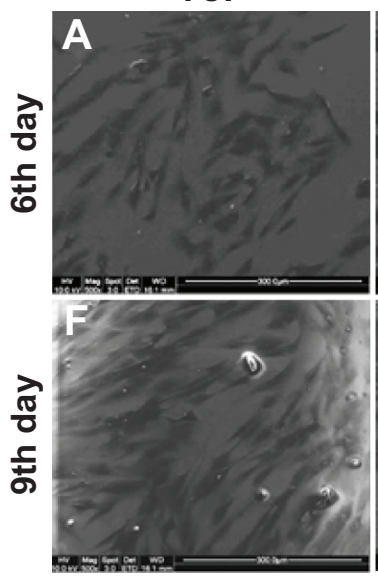

PLACL
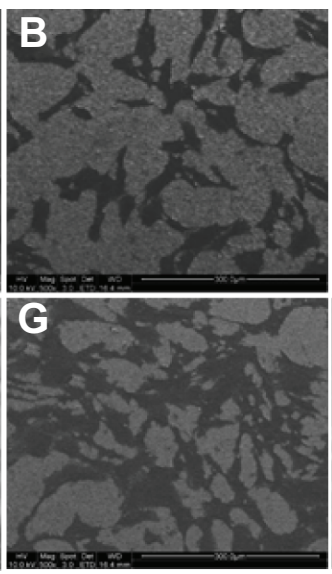

PLACL/SF
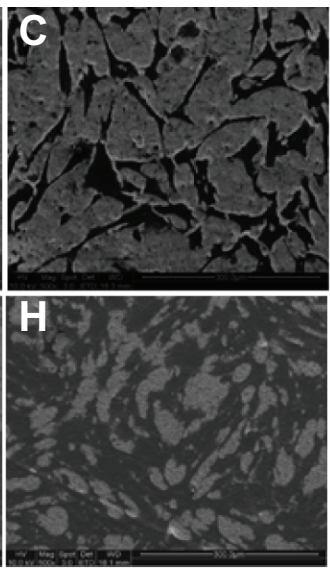

PLACL/SF/VE
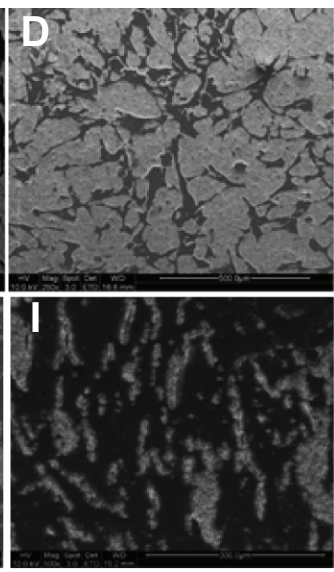

PLACL/SF/VE/Cur
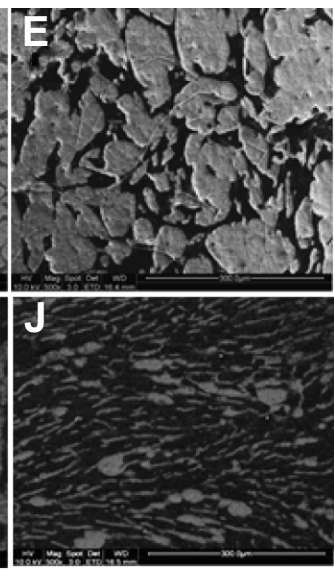

Figure 9 Scanning electron microscope (SEM) images show the cell-biomaterial interactions on day 6 (A-E) and day 9 (F-J).

Notes: (A, F) TCP, (B, G) PLACL nanofibers, (C, H) PLACL/SF nanofibers, (D, I) PLACL/SF/VE nanofibers, and (E, J) PLACL/SF/VE/Cur nanofibers at 500× magnification.

Abbreviations: PLACL, poly(L-lactic acid)-co-poly-(E-caprolactone); SF, silk fibroin; VE, Vitamin E; Cur, curcumin; TCP, tissue culture plate. 
increased on PLACL/SF/VE/Cur scaffolds (Figure 9E and J) when compared to all other nanofibrous scaffolds.

\section{Expression of CMFDA dye}

The CMFDA compound contains a group of chloromethyl derivatives established for the classification of active cells in vitro. ${ }^{45}$ The CMFDA composite is metabolized intracellularly in living cells and changed to a cell impermeant and brightly fluorescent state within 2 hours. In addition, in a reaction supposed to be facilitated by glutathione S-transferase, ${ }^{46}$ the CMFDA compound conjugates to intracellular thiols and converts to cell impermeant. The presence of the CMFDA dye was noticed to investigate the cell morphology of the scaffolds as shown in Figure 10. The cells on nanofibrous scaffolds (Figure 10B-E) formed more colonies compared to TCP (Figure 10A). In PLACL nanofibers, the number of cells was notably reduced (Figure 10B) due to the hydrophobic nature and the absence of cell binding moiety on the PLACL nanofibrous scaffolds. In PLACL/SF, PLACL/SF/ VE nanofibers (Figure 10C and D), the cell morphology was elongated, representing a good environment for cell growth due to the addition of SF and VE. We noted more cell density and normal morphology on PLACL/SF/VE/Cur nanofibrous scaffolds (Figure 10E) compared to all other scaffolds.

\section{Expression of F-actin}

Rhodamine-phalloidin staining showed the interaction of fibroblast with nanofibrous scaffolds (Figure 11). Phalloidin is a seven amino acid peptide toxin from mushroom Amanita phalloides, which binds particularly to the polymerized form of actin (F-actin) and stains the actin fibers in the cells. ${ }^{47}$ Rhodamine-phalloidin labeled actin filaments recollect many functional characteristics of unlabeled actin with their capability to interact with myosin. The cell nucleus is counterstained with DAPI (blue), Rhodamine-phalloidin staining was performed after 9 days of cell culture and F-actin stain indicated a cytoplasmic filamentous spreading under laser scanning confocal microscopy (Figure 11). It was observed that the expression of F-actin was increased on PLACL/SF/ VE/Cur (Figure 11J-L) when compared to PLACL, PLACL/ SF, and PLACL/SF/VE (Figure 11D-I). The spindle-shaped morphology of the fibroblast was observed on PLACL/SF/VE/ Cur similar to its phenotype on PLACL/SF and PLACL/SF/ VE nanofibrous scaffolds. We observed the higher cell density and better morphology on PLACL/SF/VE/Cur when compared to PLACL and PLACL/SF/VE nanofibrous scaffolds. DAPI staining indicated the higher cell density (blue dot) on PLACL/ SF/VE/Cur (Figure 11J) when compared to PLACL, PLACL/ $\mathrm{SF}$, and PLACL/SF/VE nanofibrous scaffolds (Figure 11D,
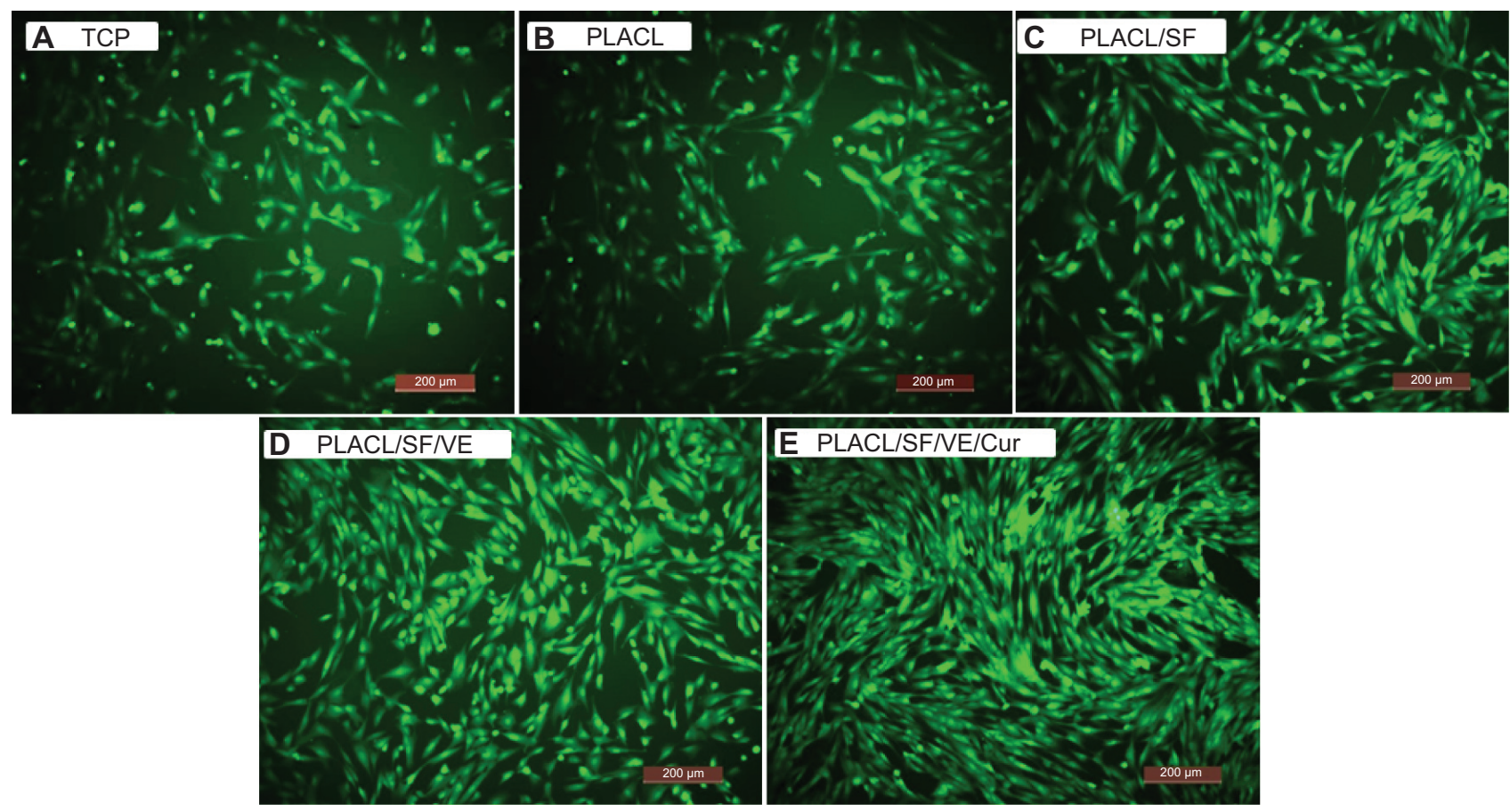

Figure 10 CMFDA dye extrusion image to analyze cell morphology on (A) TCP, (B) PLACL nanofibers, (C) PLACL/SF nanofibers, (D) PLACL/SF/VE nanofibers, and (E) PLACL/SF/VE/Cur nanofibrous scaffolds at I0× magnifications (scale bar $200 \mu \mathrm{m}$ ).

Abbreviations: PLACL, poly(L-lactic acid)-co-poly-( $\varepsilon$-caprolactone); SF, silk fibroin; VE, Vitamin E; Cur, curcumin; CMFDA, 5-chloromethylfluorescein diacetate; TCP, tissue culture plate. 

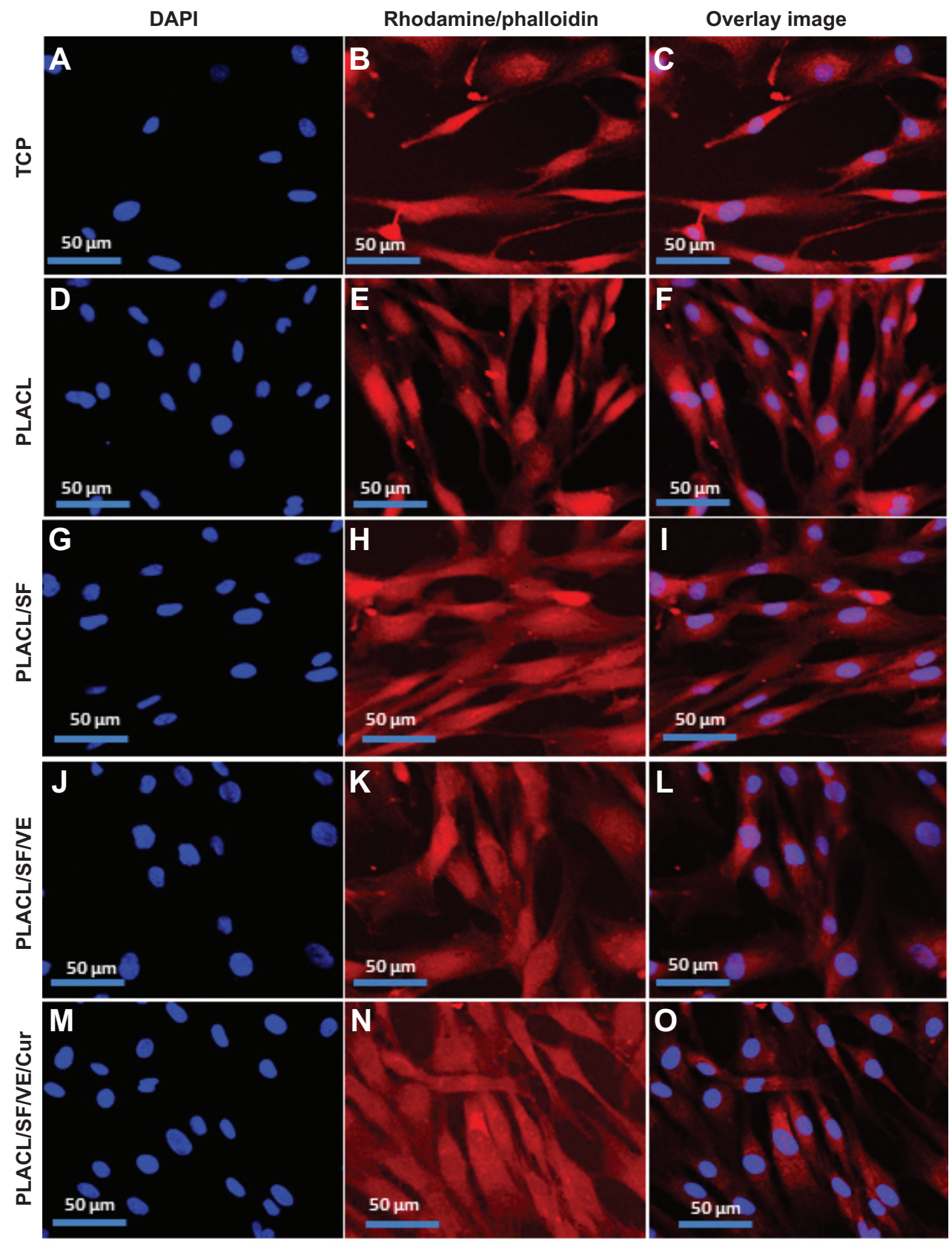

Figure I I Laser scanning confocal microscopy images.

Notes: Immunocytochemical analysis for the expression of F-actin staining with rhodamine-phalloidin (B, E, H, K, N) and (C, F, I, L, O) overlay images of (B, E, H, K, N) on TCP (B, C), PLACL (E, F), PLACL/SF (H, I) PLACL/SF/VE (K, L), and PLACL/SF/VE/Cur (N, O) with the nuclear staining by DAPI (blue fluorescence) at 20× magnification. Nucleus stained with DAPI (A, D, G, J, M).

Abbreviations: PLACL, poly(L-lactic acid)-co-poly-( $\varepsilon$-caprolactone); SF, silk fibroin; VE, Vitamin E; Cur, curcumin; TCP, tissue culture plate; DAPI, 4',6-Diamidino-2-phenylindole.

G). The obtained results showed that the biocompatibility of PLACL/SF/VE/Cur nanofibrous scaffold has promising potentiality for skin tissue regeneration.

\section{Conclusion}

Wound healing is a complex phenomenon connecting interactions between the dermal and epidermal cells with ECM. Perfect wound dressing applicants have to mimic the structure and biological role of native ECM proteins, which give support and regulate cellular activities. The interconnecting porous nanofibrous scaffold provided more structural space for the adhesion and proliferation of human dermal fibroblast and permitted efficient exchange of nutrients and metabolic wastes. The importance of this study is the application of bioactive macromolecules of SF/VE/Cur introduced into the polymeric nanofibers to improve specific biological 
functions like adhesion, proliferation, and collagen secretion for skin tissue regeneration. The incorporation of Cur and VE significantly strengthen the scaffold properties compared to PLACL/SF nanofibers. Furthermore, electrospun biocomposite porous PLACL/SF/VE/Cur nanofibrous scaffolds have good mechanical properties and biocompatibility, and are nontoxic to the surrounding tissue, and the present drug release study indicates that the Cur released from PLACL/ $\mathrm{SF} / \mathrm{VE} / \mathrm{Cur}$ nanofibrous mat in a sustained way helps to stimulate fibroblast proliferation and collagen secretion that significantly affect the wound healing properties. Human dermal fibroblasts cultured on PLACL/SF/VE/Cur composite nanofibrous scaffolds hold great potential for cellular activities ranging from cell adhesion and proliferation to collagen secretion, and help support the sustained, localized delivery of biomolecules to the site of interest so as to accelerate wound healing in skin tissue regeneration.

\section{Acknowledgments}

This work was supported in whole or in part by the Ministry of Education Start-up Grant (Srinivasan Dinesh Kumar), Lee Kong Chian School of Medicine, Nanyang Technological University, Singapore and NRF Technion (R-398-001-065592) Grant, NUSNNI, National University of Singapore.

\section{Disclosure}

The authors report no conflicts of interest in this work.

\section{References}

1. Auger FA, Lacroixd D, Germain L. Germain. Skin substitutes and wound healing. Skin Pharmacol Physiol. 2009;22:94-102.

2. Macneil S. Progress and opportunities for tissue engineered skin. Nature. 2007;445:874-880.

3. Ma L, Gao CY, Mao ZW, Shen JC, Hu XQ. Collagen/chitosan porous scaffolds with improved biostability for skin tissue engineering. Biomaterials. 2003;24:4833-4841.

4. Bottcher-Haberzeth B, Biedermann T, Reichmann E. Tissue engineering of skin. Burns. 2010;36:450-460.

5. Vander VC, van der Wal MBA, van Leeuwen MCE, Ulrich MMW, Middelkoop E. Biological background of dermal substitutes. Burns. 2010;36:305-321.

6. Ma PX, Zhang R. Synthetic nano-scale fibrous extracellular matrix. J Biomed Mater Res. 1999;46:60-72.

7. MukherjeeS, Venugopal J, Ravichandran R, Ramakrishna S, Raghunath M. Evaluation of the biocompatibility of PLACL/collagen nanostructured matrices with cardiomyocytes as a model for the regeneration of infarcted myocardium. Adv Funct Mater. 2011;21(12):2291-2300.

8. Nejati E, Mirzadeh H, Zandi M. Synthesis and characterization of nanohydroxyapatite rods/poly(L-lactide acid) composite scaffolds for bone tissue engineering. Composites Part A. 2008;39:1589-1596.

9. Webster TJ, Ergun C, Siegel RW, Bizios R. Specific proteins mediate enhanced osteoblast adhesion on nanophase ceramics. J Biomed Mater Res. 2000;51:475-483.

10. Webster TJ, Siegel RW, Bizios R. Osteoblast adhesion on nanophase ceramics. Biomaterials. 1999;20:1221-1227.
11. Sobajo C, Behzad F, Yuan XF, Bayat A. A potential medium for tissue engineering. Eplasty. 2008;8:438-446.

12. Roh DH, Kang SY, Kim JY, et al. Wound healing effect of silk fibroin/ alginateblended sponge in full thickness skin defect of rat. J Mater Sci Mater Med. 2006;17(6):547-552.

13. Daithankar AV, Padamwar MN, Pisal SS, Paradkar AR, Mahadik KR. Moisturizing efficiency of silk protein hydrolysate: silk fibroin. Indian J Biotechnol. 2005;4:115-121.

14. Vepari C, Kaplan DL. Silk as a biomaterial. Prog Polym Sci. 2007; 32: 991-1007.

15. Bhardwaj N, Kundu SC. Electrospinning. A fascinating fiber fabrication technique. Biotechnol Adv. 2010;28:325-347.

16. Lu P, Ding B. “Applications of electrospun fibers," Recent patents on nanotechnology. Recent Pat Nanotechnol. 2008;2:169-182.

17. Li M, Wu Z, Zhang C, et al. Study on porous silk fibroin materials. II. Preparation and characteristics of spongy porous silk fibroinmaterials. J Appl Polym Sci. 2001;79(12):2192-2199.

18. Yang Wang C, Zhang K, Yi Fan K, Mo X, Ruan H, Li F. Aligned naturalsynthetic polyblend nanofibers for peripheral nerve regeneration. Acta Biomater. 2011;7:634-643.

19. Fan LP, Wang HS, Zhang KH, et al. Vitamin C-reinforcing silk fibroin nanofibrous matrices for skin care application. RSC Adv. 2012; 2:4110-4119.

20. Hosseini Ravandi SA, Gandhimathi C, Valizadeh M, Ramakrishna.S Application of electrospun natural biopolymer nanofibers. Curr Nanosci. 2013;11:423-433.

21. Min BM, Jeong L, Nam YS, Kim JM, Kim JY, Park WH. Formation of silk fibroin matrices with different texture and its cellular response to normal human keratinocytes. Int J Biol Macromol. 2004;34:223-230.

22. Trivedi JS, Krill SL, Fort JJ. Vitamin E as a human skin penetration enhancer. Eur J Pharm Sci. 1995;3:241-243.

23. Thiele JJ, Ekanayake-Mudiyanselage S. Vitamin E in human skin: organ-specific physiology and considerations for its use in dermatology. Mol Aspects Med. 2007;28:646-667.

24. Liang G, Li X, Chen L, et al. Synthesis and anti-inflammatory activities of mono-carbonyl analogues of curcumin. Bioorg Med Chem Lett. 2008; 18:1525-1529.

25. Xiaoyue S, Linpeng F, Chuanglong H, Kuihua Z, Xiumei M, Hongsheng W. Vitamin E-loaded silk fibroin nanofibrous mats fabricated by green process for skin care application. Int J Biol Macromol. 2013;56:49-56.

26. Juan P, Teresa C, Isabel IB, Antonio LD, Marıa VR. Long-term degradation of poly(3-caprolactone) films in biologically related fluids. Polym Degrad Stab. 2006;91:1424-1432.

27. Ravichandran R, Liao S, Ng CH, Chan CK, Raghunath M, Ramakrishna S. Effects of nanotopography on stem cell phenotypes. World J Stem Cells. 2009;1:55-56.

28. Ravichandran R, Venugopal JR, Sundarrajan S, Mukherjee S, Ramakrishna S. Poly(glycerol sebacate)/gelatin core/shell fibrous structure for regeneration of myocardial infarction. Tissue Eng Part A. 2010;17:1363-1373.

29. Venugopal J, Zhang YZ, Ramakrishna S. Biomimetic surface modification of titanium surfaces for early cell capture by advanced electrospinning. Artif Organs. 2006;30(6):438-444.

30. Yoo CR, Yeo IS, Park KE, et al. Effect of chitin/silk fibroin nanofibrous bicomponent structures on interaction with human epidermal keratinocytes. Int J Biol Macromol. 2008;42:324-334.

31. Ratner BD, Hoffman AS, Schoen FJ, Lemons JE. Biomaterials Science: An Introduction to Materials in Medicine. 2nd ed. Amsterdam: Elsevier; 2004.

32. Gandhimathi C, Venugopal J, Ravichandran R, Sundarrajan S, Suganya S, Ramakrishna S. Mimicking nanofibrous hybrid bone substitute for mesenchymal stem cells differentiation into osteogenesis. Macromol Biosci. 2013;13:696-706.

33. Ricci G, Patrizi A, Bendandi B, Menna G, Varotti E, Masi M. Clinical effectiveness of a silk fabric in the treatment of atopic dermatitis. Br J Dermatol. 2004;150:127-131. 
34. Gupta D, Venugopal J, Mitra S, Giri Dev VR, Ramakrishna S. Nanostructured biocomposite substrates by electrospinning and electrospraying for the mineralization of osteoblasts. Biomaterials. 2009; 30:2085-2094.

35. Martinez EC, Ivirico JL, Criado MI, Ribelles G, Pradas MM, Sanchez MS. Effect of poly (L-lactide) surface topography on the morphology of in vitro cultured human articular chondrocytes. J Mater Sci Mater Med. 2007; 18:1627-1632.

36. Kwideok P, Young MJ, Jun SS, Kwang-Duk A, Dong KH. Surface modification of biodegradable electrospun nanofibre scaffolds and their interaction with fibroblast. J Biomater Sci Polym Ed. 2007;18:369-382.

37. Zahedi P, Rezaeian I, Ranaei-Siadat SO, Jafari SH, Supaphol P. A review on wound dressings with an emphasis on electrospun nanofibrous polymeric bandages. Polym Adv Technol. 2010;21:77-95.

38. Agrawal CM, Ray RB. Biodegradable polymeric scaffolds for musculoskeletal tissue engineering. J Biomed Mater Res. 2001;55:141-150.

39. Giesa T, Arslan M, Pugno NM, Buehler MJ. Nanoconfinement of spider silk fibrils begets superior strength. Extensibility and toughness. Nano Lett. 2011;11:5038-5046.

40. Ignatious F, Baldoni JM, inventor; Smikthkline Beecham Corporation, assignee. Electrospun pharmaceutical compositions. United States patent PCT/US01/02399. 2001 Jan 25.
41. Van Kooten TG, Whitesides JF, von Recum AF. Influence of silicon (PDMS) surface texture on human skin fibroblast proliferation as determined by cell cycle analysis. J Biomed Mater Res. 1998;43:1-14.

42. Barnes MJ, Morton LF, Bennett RC, Bailey AJ, Sims TJ. Fabrication of nanostructured hydroxyapatite and analysis of human osteoblastic cellular response. Biochem J. 1976;157:263-266.

43. Kim E, Tsenguun B, Shin EJ, Chang KH. Fibrin derived peptides stimulate glucose transport in normal and insulin resistant 3T3-L1 adipocytes. Biol Pharm Bull. 2009;32:427-433.

44. Guo X, Gough JE, Xiao P, Liu J, Shen ZF. Fabrication of nanostructured hydroxyapatite and analysis of human osteoblastic cellular response. J Biomed Mater Res. 2007;82:1022-1032.

45. Zhang YZ, Olson N, Mao F, Roth B, Haugland RP. New fluorescent probes for long-term tracing of living cells. FASEB J. 1992;6:1835.

46. Zhang YZ, Naleway JJ, Larison KD, Huang ZJ, Haugland RP. Detecting lacZ gene expression in living cells with new lipophillic fluorogenic beta-galactosidase substrates. FASEB J. 1991;5:3108-3113.

47. Cooper J. Effects of cytochalasin and phalloidin on actin. J Cell Biol. 1987; 105:1473-1478.
International Journal of Nanomedicine

\section{Publish your work in this journal}

The International Journal of Nanomedicine is an international, peerreviewed journal focusing on the application of nanotechnology in diagnostics, therapeutics, and drug delivery systems throughout the biomedical field. This journal is indexed on PubMed Central, MedLine, CAS, SciSearch ${ }^{\circledR}$, Current Contents ${ }^{\circledR} /$ Clinical Medicine,

\section{Dovepress}

Journal Citation Reports/Science Edition, EMBase, Scopus and the Elsevier Bibliographic databases. The manuscript management system is completely online and includes a very quick and fair peer-review system, which is all easy to use. Visit http://www.dovepress.com/ testimonials.php to read real quotes from published authors. 